\title{
Article
}

\section{Using Flipgrid to Improve Reflection: A Collaborative Online Approach to Coach Development}

Stoszkowski, John Robert, Hodgkinson, Aran and Collins, David John Available at http://clok.uclan.ac.uk/33808/

Stoszkowski, John Robert ORCID: 0000-0002-1968-5770, Hodgkinson, Aran ORCID: 0000-0002-2310-5804 and Collins, David John ORCID: 0000-00027601-0454 (2021) Using Flipgrid to Improve Reflection: A Collaborative Online Approach to Coach Development. Physical Education and Sport Pedagogy, 26 (2). pp. 167-178. ISSN 1740-8989

It is advisable to refer to the publisher's version if you intend to cite from the work. http://dx.doi.org/10.1080/17408989.2020.1789575

For more information about UCLan's research in this area go to http://www.uclan.ac.uk/researchgroups/ and search for <name of research Group>.

For information about Research generally at UCLan please go to http://www.uclan.ac.uk/research/

All outputs in CLoK are protected by Intellectual Property Rights law, including Copyright law. Copyright, IPR and Moral Rights for the works on this site are retained by the individual authors and/or other copyright owners. Terms and conditions for use of this material are defined in the policies page.

\section{CLoK}

Central Lancashire online Knowledge www.clok.uclan.ac.uk

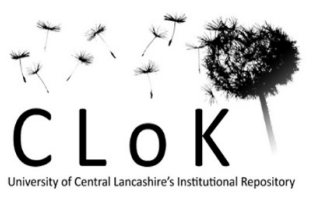




\section{Using Flipgrid to Improve Reflection: A Collaborative Online Approach to Coach Development}

John Stoszkowski $^{\mathrm{a}^{*}}$, Aran Hodgkinson ${ }^{\mathrm{a}}$ and Dave Collins ${ }^{\mathrm{b}, \mathrm{c}}$

${ }^{a}$ School of Sport and Health Sciences, University of Central Lancashire, Preston, England, UK.

${ }^{b}$ Moray House School of Education and Sport, University of Edinburgh, Edinburgh, Scotland, UK.

${ }^{c}$ Grey Matters Performance Ltd. Stratford upon Avon, England, UK.

*Corresponding author. School of Sport and Health Sciences. University of Central Lancashire, Preston, PR1 2HE, UK. Email: JRStoszkowski@uclan.ac.uk

Date of original submission: $29^{\text {th }}$ November 2019

Date of revised submission: $25^{\text {th }}$ April 2020

Date of final submission: $22^{\text {nd }}$ June 2020

Word count (inclusive of tables/references): 7000 


\section{Using Flipgrid to Improve Reflection: A Collaborative Online Approach to Coach Development}

Background: Coaching researchers have stressed the need for critical reflection in practitioners, as an important tool to facilitate development. In parallel, however, coaches' preference for more informal development methods are well documented. The increased impact of social media and other online applications offers new ways to pursue these important factors, and previous research has explored the use of written online blogs as a medium for this purpose.

Purpose: The objective of the current study was to explore the potential effectiveness of Flipgrid, a video-based online communication tool that enables face-to-face, short verbal interactions, for facilitating and promoting collaborative online learning and critical reflection.

Research design and data collection: The intervention was applied to a group of student-coaches ( $n$ $=21$ ), enrolled in an undergraduate sports coaching degree programme at a higher education institution in the UK, as a part of their educational diet. Responses were analysed using Hatton and Smith's (1995) reflective framework; an approach used previously in coach development studies. Quantitative and qualitative data were collected reflecting the number and reflective level of interactions over a fifteen-week period.

Findings: Results showed good support for the approach, with participants exhibiting more frequent and more critical responses as compared to earlier studies which had used a blog-based written response and interaction format.

Conclusion: Further research is worthwhile to examine the broader usage of the approach. However, in this higher education setting, the approach proved impactful with these student-coaches.

Keywords: coach education; coach learning; reflective practice; social learning 


\section{Summary for Practitioners}

Critical reflection, thinking through ideas and comparing methods against research and practice, is an important part of professional development. This is particularly true of coaching. Therefore, coach educators have looked at a variety of approaches and tools which can be used to encourage and facilitate this process. Such a search should also take advantage of the increasing use and importance of social media, especially for younger people. Accordingly, this preliminary investigation used Flipgrid, an online video discussion platform, to facilitate critical reflection and verbal debate with a sample of 21 undergraduate sport coaching students. Use of this application was associated with a greater number and deeper level of critical interactions, when contrasted with a previous study which had used blog-based written interactions. Using verbal interactions would appear to facilitate interactions for 'Generation Z' students, making the application very suitable for use, especially with younger coaches. 


\section{Introduction}

In recent years, the rapid expansion of internet access and the dramatic increase in ownership of mobile technologies (e.g., laptops, tablets and smartphones) has fundamentally transformed the way people work, communicate and have access to information (Tsiotakis and Jimoyiannis 2016). As a natural consequence, this has also driven significant changes in when, where, and how people learn (Courtney and Wilhoite-Mathews 2015), or at least in their felt preferences for certain approaches over others. Another, albeit parallel preference, driven in part by social constructivist learning assumptions, is the apparent low impact of formal coach education initiatives on coach learning and the suggestion that coaches prefer to learn informally with their peers (Stoszkowski and Collins 2016). As a consequence of these preferences, the provision of technology-enhanced and online collaborative learning experiences to facilitate and enhance coach learning and development has grown rapidly (Cushion and Townsend 2019). Web 2.0 tools and platforms (e.g., blogs, forums, wikis, social networking sites, e-portfolios) are relatively easy to use and cheap to access, and are said to enable and promote social interaction, sharing and the co-construction of knowledge among people with common interests (Byington 2011). As such, Web 2.0 tools have been viewed as ripe for exploitation in coach education (Piggott 2015) and they have seen dynamic growth as both a compliment and alternative to traditional face-to-face formal coach education courses and certifications (Cushion and Townsend 2019).

Nevertheless, the effectiveness of these online tools and applications for facilitating collaborative learning and social interaction is still somewhat debatable, whilst our understanding of the learning processes involved are still evolving (Tsiotakis and Jimoyiannis 2016). Questions remain as to how we can best design and operate online learning environments to ensure coach educators not only meet the espoused wants and expectations of coaches, but their actual needs as well. For example, peer discussion is more than just that, 
and coaches (and, therefore, coach educators) need to be aware of the importance and value of activities like structured critical reflection in order for maximum benefit to be garnered from their coaches' social learning experiences (Stoszkowski and Collins 2012). Furthermore, collaborative and critically reflective online coach discussion, grounded in applied practice and theoretical concepts, does not occur automatically and requires a significant amount of scaffolding and 'up front' priming work by instructors (Resta and Laferrière 2007), as well as active coordination of group dynamics (Kreijins, Kirschner, and Jochems 2003). Even once this essential groundwork has been completed, and research suggests that this is far from being a simple task, other challenges need to be overcome. For example, the creation of stimulating learning environments that encourage productive interactions between participants requires the careful matching of technological tools with modern pedagogy and fundamental principles derived from cognitive, educational, and social science research (Abrami et al. 2011).

One tool that has been tested in coach education for its potential to strengthen and promote critical thinking and collaborative reflection on professional practice is the use of online blogs (Stoszkowski and Collins 2014; Stoszkowski and Collins 2017). Although Stoszkowski and Collins (2017) concluded that online blogs were a useful tool to promote higher-order reflective thinking and fully-functioning communities of practice (Wenger, McDermott, and Snyder 2002), and student-coaches were generally very positive about their learning experiences when using them (Stoszkowski, Collins, and Olsson 2017), questions remained over the extent to which the predominantly text-based medium used in most contemporary online learning environments was the most appropriate means to support the tasks that participants need to perform (Sins et al. 2011). For example, reflective discussion involves trying to move from a state of perplexity, ambiguity, obscurity, conflict, doubt, and incoherence toward a clarified and coherent situation (Clarà 2015). Yet, tools and platforms 
that rely on text-based communication unavoidably restrict the exchange of auditory, visual and nonverbal communication cues that help people regulate interactions, convey ideas and monitor feedback from others (Straus 1997). Similarly, the prerequisite social interaction that is needed to facilitate dialogue that leads to deep learning and information retention (Van der Linden and Renshaw 2001) can become unusually complex in text-based environments (Gunawardena 1995; Northrup 2001). Finally, as shown by Stoszkowski and Collins (2018), and reflecting our earlier comments of the need for essential groundwork, these approaches require both a level of knowledge/maturity and some intrinsic drive in the participants to ensure an appropriate commitment and involvement (Abrami et al. 2011). In short, whilst the approaches show promise, there are still some hurdles to be overcome if general benefits are to accrue.

Prior to the advent and widespread use of Web 2.0 technologies, McGrath and Hollingshead (1993) proposed the 'task-media fit' hypothesis as a framework to predict the effect of different communication media on performance for different task types. This theory states that the effectiveness of a communication mode for a given task depends on the degree of fit between the richness of information that can be transmitted via that tool or application and the information richness requirements of that task (Sins et al. 2011). The richness of a medium is said to depend on four main attributes: (1) the immediacy of feedback; (2) the number of cues and channels available; (3) language variety; and (4) the degree to which intent is focused on the receiver. By these criteria, face-to-face communication is considered the 'richest' medium as people can quickly convey information and meaning both verbally and nonverbally. In contrast, communication reliant on the conveying of 'mere' textual information (such as in an online blog) is said to be low in media richness (Sins et al. 2011). When accomplishing tasks such as decision making, learners typically rate text-based, computer-mediated communication as low in media richness (Kreijins, Kirschner, and 
Jochems 2003). This was found to be so because it can constrain vocabulary and reduce the potential for expressive communication through cues that are transmitted through vision (e.g., facial expressions, posture, gaze and gestures) or sound (e.g., voice volume, inflection and tone). All this quite apart from the inherent limitations on speed for all but the most talented typists.

Furthermore, tasks that require groups to 'negotiate and resolve conflicts of views or conflicts of interests may require the transmission of maximally rich information' (McGrath and Hollingshead 1993, 92). As such, tools and platforms that provide less richness of information than the task requires may constrain communication such that the media is incapable of transmitting the types of or amount of communication needed to effectively address the task (Mennecke, Valacich, and Wheeler 2000). Effectual collaborative learning and critical reflection require participants to apply reflective processes that go beyond superficial and descriptive activities (Thompson and Pascal 2012). As part of this, the identification and critique of any assumptions that are being made, as well as the values and beliefs that are being drawn upon, is required (Saylor 1990). Consequently, the task-media fit hypothesis would predict that a rich mode of communication is required. Accordingly, the aim of the current study was to explore the potential effectiveness of Flipgrid for promoting collaborative online learning and critical reflection. Our expectation was that the face-to-face verbal communication involved in this recent application would generate increased quality of interaction across a sample of trainee coaches.

\section{Flipgrid}

Flipgrid (http://www.flipgrid.com/) is an asynchronous online video discussion platform that allows the sharing of user-generated videos in a 'social learning community'. It can be used with a web-based browser on a Mac or PC as well as via the native Flipgrid iOS or Android 'app' on mobile devices (i.e., a smartphone or tablet). The account holder creates a 'grid' 
then invites users to respond verbally to 'topics' (i.e., questions or prompts) with a video recording. Grids, which essentially become collections of topics, are managed through an easy to navigate 'educator dashboard'. When creating a new grid or topic, the account holder can customize several elements including response moderation, privacy settings, and the extent to which users can add stickers and drawings to their videos. To start a discussion, the account holder simply creates a new topic. Video and/or images can be included in the initial prompt as well as an external link to other online resources (e.g., an article or cloud-based document). To share a grid with users, the account holder can distribute a custom URL that will take users directly to the grid where they can click on and respond to a topic.

Alternatively, the account holder can send those using the iOS or Android app a grid code that they simply type in and use to access the grid and its contained topics. Users enter a name and email address, but they are not required to create their own account to use the platform. Grids are private and can only be accessed by those who have the relevant link or code, the account holder can also elect to password-protect access if so desired.

The user interface is intuitive and functions similarly to many other popular social media platforms (e.g., Instagram and Snapchat). To record a video response to a topic, or reply to another user's response, users record video using Flipgrid's easy to use video recorder on their Internet-connected computer or mobile device (web camera and microphone required). Users can pause and rerecord their video as many times as they wish before it is uploaded to the grid, where it can then be viewed by peers who have logged in to the site. All responses and replies are displayed linearly in date order and they can be watched individually or as a slide show. Users can elect to 'follow' a grid and receive email notifications whenever new content is uploaded. Flipgrid automatically tracks and records discussion statistics and user engagement (e.g., date and time of videos; the number of responses and replies; the number of views for each reply or response; and the total 
engagement time in hours of recorded video), which can be downloaded in spreadsheet format. Flipgrid has a closed-captioning feature for accessibility and audio transcripts can be downloaded as text documents, the Flipgrid website also offers detailed tutorials and technical support.

At the time of data collection during the current study, the Flipgrid licence cost $\$ 65$ for a one-year subscription; this allowed the account holder to create an unlimited number of grids and topics and limit the response length of videos from 30 seconds up to a maximum of 5 minutes. The account holder could also create custom rubrics to allow them to provide written and/or video-based feedback on a user response that only that user can see. A free version of Flipgrid was also available; however, it limited account holders to one grid with unlimited topics, the length of responses was restricted to either 15 or 90 seconds, and responders could only reply to the initial topic, not each other. In late 2018, Flipgrid was acquired by Microsoft and is now available free of charge to anyone in education with an Office365 account.

\section{Method}

\section{Participants}

The sample in the present study consisted of twenty-one final-year undergraduate studentcoaches (5 females and 16 males) enrolled in a BA (Hons.) sports coaching degree programme during the 2018 academic year. The average age of the participants was 21.33 years $(\mathrm{SD}=0.91)$ and the median coaching experience was reported as 3.67 years, with experience ranging from 2 to 6 years in a variety of sports (See Table 1). All participants had completed at least one national governing body coaching award, with the highest awarded qualification translating to level 2 of the UK coaching certificate endorsed framework (Sports Coach UK 2012). As part of their programme, all participants were undertaking a selfsourced work placement incorporating a minimum of 40 hours coaching practice, as well as 
concurrently coaching in the community in a variety of paid and voluntary roles (i.e., over and above any practical coaching linked to their programme of study). This was to be followed by a more formal reflection on the lessons learned, and it was this element on which our investigation was focused.

In anticipation, ethical approval was obtained from the authors' institutional ethics committee. Then, on completion of the placement, all student-coaches on the module were then emailed an invite to participate in the study, which included an information sheet that outlined the purpose of the study and appropriate ethical considerations (e.g., declaration of confidentiality, right of withdrawal). It was explained that participation in the research project was voluntary and assurances were made that participation would not impact on their module grade in any way. If the student-coaches wished to take part, they were asked to provide their informed consent via return email, which they all did.

\section{Procedures}

The module in question, entitled 'coaching practice and reflection', was a self-selected optional module that ran for 30 weeks of the academic year. For the duration of the module, student-coaches were responsible for completing a work-based placement in a community coaching setting of their own arrangement. During the second semester of the module (15 weeks), and consistent with the protocol outlined by Stoszkowski and Collins (2017), the student-coaches were encouraged to collaboratively acquire knowledge and skills by sharing, discussing, and reflecting upon their on-going learning and practical experiences in relation to a series of themes.

Building upon the protocol established by Stoszkowski and Collins (2017), whereby the text based blogging platform WordPress provided a learning stimulus together with a basis for evaluation, in the present study Flipgrid provided the main teaching and learning environment with three separate 'grids' (one grid with 6 members, one grid with 8 members, 
one grid with 7 members) set up. Each grid was private and could only be viewed by its members and two module tutors. In an initial workshop, the module handbook, intended learning outcomes, and associated assessment criteria for the module were outlined. Then, the conceptual purpose and potential educational value of collaborative learning (Stoszkowski and Collins 2017) was explained and explored. A follow up workshop was split into two. In the first half, the module tutors (who were both full-time academics with at least 10 years' experience) introduced the principles, value, and purpose of critical reflection. Here, Thompson and Pascal's (2012) theoretical base was presented as an exemplar framework to guide the reflective process. The second half then focused on Flipgrid and gave an overview and demonstration of the platform. In the second week of the semester, the first of three workshops focussing on separate 'themes' took place. The first two themes were (1) coach development and (2) shared leadership and athlete empowerment. The third theme was openended in that it did not have an explicit topic focus. Instead, it required student-coaches to state, explain, and justify a specific view they held in relation to coaching, with the other members of their group then tasked with attempting to change that view. Each workshop was interactive and involved tutor-facilitated discussion and debate, the aim of which was to encourage student-coaches to question their previous assumptions and provide an initial stimulus and knowledge foundation upon which to base their subsequent online discussions that took place between each workshop. Each workshop was separated by an average period of four weeks for the remainder of the semester, with 'topics' that related to each theme set up by the two module tutors on each grid. The time limit for videos was set at 150 s. Each student-coach's module grade was based on their individual participation in their group's Flipgrid grid (i.e., the quality and quantity of their responses and replies to topics).

\section{Data Analysis}

Descriptive content analysis was used to examine each grid in terms of the number of 
videos (i.e., responses and replies), the frequency of entries, the length of each video and the number of views each video received. This represented student-coaches' participation and engagement. The audio transcript for each video was then downloaded and transferred to a Microsoft Word document. The first author watched each video in order to identify and correct any errors in the automated transcription. Then, a grid-by-grid content analysis of all video transcripts was conducted by the second author in order to examine the reflective quality of the comments posted. Each transcript was coded in line with Hatton and Smith's (1995) reflective framework, which had already been used successfully to identify levels of reflection in blog entries (Stoszkowski and Collins 2014; Stoszkowski and Collins 2017). Although the framework was originally designed to assess reflective writing, the categories it contains lend themselves equally to assessing the reflective quality of videos once they have been transcribed. The framework includes four levels, rising in ascending order of reflective quality: unreflective description (i.e., basic descriptions of events with no attempt to provide reason or justification), descriptive reflection (i.e., attempts to provide reasons for events or actions but reported in a descriptive way), dialogic reflection (i.e., more analytical reflection that involves stepping back from and exploring reasons for events, whilst evidencing attempts to appreciate wider contexts and alternative points of view) and critical reflection (i.e., awareness that actions and events are not only explicable by multiple perspectives, but are also located in and influenced by multiple historical, cultural and sociopolitical contexts). Each transcript was coded according to the highest level of reflection reached in that video (Freeman and Brett 2012).

During the above content analysis, the first and third author would periodically check the assigned categories for each transcript segment (roughly every $10^{\text {th }}$ video). On the occasions where the three authors disagreed about the category in which a video was placed (less than $5 \%$ of entries), negotiation was pursued until a consensus of opinion was reached. 
Then, in line with the procedures followed by Stoszkowski and Collins (2017), a reliability check was conducted by an independent investigator, trained in qualitative methodology but blind to the objectives of the study, who audited the assigned categories to ensure that they accurately reflected the video transcripts. This process attempted to avoid the researchers' own subjectivities displacing the reflections of the participants, whilst discussing the decisions made (Nowell et al. 2017). To further ensure credibility, transparency, and trustworthiness, the second author maintained a reflexive diary throughout the data analysis process whereby the coding for each video was given a positioning statement to allow the independent investigator to challenge, understand, and review the assigned code effectively (Smith and McGannon 2018). No issues were found.

\section{Results}

A total of 571 Flipgrid videos were analysed. Table 2 and Table 3 show that the participants in each Flipgrid group actively engaged with one another by posting videos for the duration of the semester (i.e., for all three themes), with the number of videos posted by each participant ranging from 6 to $69(\mathrm{M}=26.58, \mathrm{SD}=15.42)$, and the number of videos per group ranging from 113 to $230(\mathrm{M}=190.33, \mathrm{SD}=66.98)$. Videos ranged from a minimum of 15 seconds to a maximum of 150 seconds in length $(M=111, M d n=117, S D=24)$. Tutors uploaded a total of 24 videos across all three groups during the semester, replying to videos an average of three times per theme on each group's grid. These videos were largely encouraging in nature (i.e., highlighting a well-made point) or intended to prompt further discussion by posing a question or identifying a missed opportunity for further exploration.

A total of 543 videos were coded in line with Hatton and Smith's (1995) reflective framework. This analysis excluded 28 videos that had previously been coded as 'acknowledgement' or 'misdirected entry' (see Table 2) due to their short length and/or inapplicable content (e.g., a video mistakenly uploaded too soon or a video simply providing 
additional information such as a reference for an earlier video). Table 3 shows that the vast majority of videos were coded at the third 'level' of Hatton and Smith's (1995) reflective framework, with $78.60 \%$ of entries constituting dialogic reflection. No videos were coded as unreflective description (the lowest level of the framework), $15.83 \%$ of entries constituted descriptive reflection and the number of entries coded as critical reflection was $5.57 \%$ (the highest level of the framework). Although some variability in levels of reflection was evident between individual participants and between groups, the majority of participants were able to reach the dialogic reflection level. For example, of the 21 participants, only one failed to post a video coded as dialogic reflection. However, most participants found it difficult to reach the critical reflection level, with only three participants posting more than one video coded as critical reflection; notably, all three of those participants were in the same group (i.e., group A, see Table 3). Table 4 provides exemplar quotes representative of each level of reflection.

\section{Discussion}

The aim of this preliminary study was to explore the extent to which Flipgrid facilitated collaborative online reflection on professional practice between groups of student-coaches, as well as how this activity compares to structured group blogging that utilises a written format. The findings suggest that the levels of reflective thinking evidenced by the majority of participants were, on average, more critical and less descriptive than those in Stoszkowski and Collins' (2017) study, which employed text-based (i.e., written) reflective group blogs. In the current study, dialogic reflection was clearly the most prominent type of reflection displayed by the participants, with a total of 427 (78.60\%) out 543 videos being coded as such. In Stoszkowski and Collins' (2017) study which utilised text-based blogs, only $48.72 \%$ of blog posts were coded as dialogic and descriptive reflection accounted for $35.17 \%$ of textbased blog posts compared to $15.83 \%$ when using Flipgrid. Unreflective description, the lowest form of reflection, was completely absent when using Flipgrid, whereas Stoszkowski 
and Collins (2017) found that unreflective description accounted for $12.20 \%$ of written blog posts. However, critical reflection only accounted for 30 videos $(5.57 \%)$ in the current study; in contrast, Stoszkowski and Collins (2017) reported $13.75 \%$ of written blog posts were coded as critical reflection.

Generally, then, these outcomes appear to suggest that Flipgrid might be a more useful tool to facilitate and compliment informal coach learning and development, which, it has been suggested, coaches prefer (e.g., Stoszkowski and Collins 2016). Notably, however, levels of critical reflection (the 'highest' category) were lower in the present, Flipgrid-driven circumstance. In this regard, we should stress that our decision to use the same reflective categorisation system was deliberate and designed to facilitate comparison against the hypothesised differences which we expected from the multisensory interactions which Flipgrid enabled. However, whilst there was clear progression in the levels of reflective thought in the present study, it is important to unpick further potential reasons for the differences with Stoszkowski and Collins' (2017) findings.

First, critical reflection is not something that comes naturally to most learners (Yang, 2009) and is often a skill which is acquired over a period of time (Leach, Neutze, and Zepke 2001). This may explain the relative lack of critical reflection in the current study when compared to text-based blogs. For example, Stoszkowski and Collins' (2017) study was carried out over 23 weeks rather than 15 weeks, which provided those student-coaches with a longer period of time to develop their reflective skills and their ability to provide rich responses. Indeed, the more asynchronous nature of written response perhaps permits learners more time to collect their thoughts and assess the information they have before responding with their opinions (Dymoke and Harrison 2008). Although the module tutors introduced the principles, value, and purpose of critical reflection (Thompson and Pascal 2012) prior to the commencement of the Flipgrid process, producing this level of reflection can often be an 'act 
of professional artistry’ (Schön 1991). Critical reflection involves an individual withdrawing themselves, stepping away from the centre of involvement, and visualising practical situations occurring in the future (Stronach, Garratt, Pearce, and Piper 2007); a difficult behaviour for individuals to master and one that requires time to develop.

Second, it may be that the verbal format provided by Flipgrid offered participants an easier process for communication but one which kept them in their 'critically-lite', comfort zone. Certainly, short sharp and electronically enabled communication is an identified characteristic of Generation Z individuals (e.g., Gould, Nalepa and Mignano 2019), which our participants indubitably were! As a result, it may have been easier, or felt more natural, for our participants to have initiated and maintained dialogic reflection (as they were immediately engaged in a familiar medium and manner of communication) but not to get into more rigorous reflective interactions.

Third, when individuals communicate face-to-face through an online platform such as Flipgrid, they are consciously framing, constructing, and presenting their 'identity' to their peers (Walker 2000), particularly when attempting to be more critical and/or sceptical of specific coaching methods and practices. Consequently, it is likely that the student-coaches in the current study were actively considering the impact of their Flipgrid responses and how they would be perceived by the other members of their group. Might it be that the more critically reflective, analytical, and philosophical a student-coaches' response is, the more likely they feel their 'identity' will be judged by peers? This might explain why critical reflection was higher in text-based blogs, as it may have felt 'easier' for the student-coaches in Stoszkowski and Collins' (2017) study to express (potentially) unpopular opinions. Similarly, we must also consider the potential impact of social anxiety (Schlenker and Leary 1982) when using video-based online tools such as Flipgrid, especially when a large percentage of the population state that they experience anxieties when speaking in front of 
others (LeFebvre, LeFebvre, and Allen 2018). It is not unreasonable, therefore, to suggest that some participants within the current study may have been overwhelmed with the face-toface communication method and this may have limited the extent of the critical reflection within their videos due to potential social discomfort (Richmond, Wrench, and McCroskey 2013).

Finally, it is commonly noted that different forms of 'scaffolding' are required when using online blogs to support student learning and promote further analytical skills (Wass, Harland, and Mercer 2011). The extent of the scaffolding applied often depends on the tutor and is considered a skill. Furthermore, the types of scaffolding the tutor decides to employ will also affect the given outcome. Considering the relative lack of critical reflection in the current study, it could be suggested that further scaffolding and input from the module tutors was required in order to 'nudge' the student coaches towards a more critically reflective level of participation. For example, more individualised support and guidance may have been needed in order to nurture critical reflection (Whipp 2003). Interestingly, the dialogic reflection exhibited in the current study developed in a linear process, with each group increasing the amount of dialogic reflection they achieved during each theme. However, did this trend occur because the student-coaches became more familiar with the processes of the face-to-face blog, or because they gradually acquired and bought into the educational intentions and purpose of the tutors? The participants did not have any previous experience with this style of learning (in a formal setting at least), and instead were much more familiar with traditional delivery methods and cognitive assurance (Wass, Harland and Mercer 2011). However, as the process evolved, it appears that the student-coaches' view of knowledge as incontestable moved toward a greater comfort with epistemological uncertainty (cf. Entwistle and Peterson 2004), which resulted in them becoming more comfortable questioning, analysing, and challenging their peers' views. 


\section{Conclusion}

This preliminary investigation has offered some generally positive data to support the inclusion of Flipgrid as a teaching and learning tool for coaches in training. As described, use of the same categorisation analysis as earlier papers has enabled us to demonstrate greater reflective thought and interaction across the group, albeit that the highest category was more apparent in written rather than the present, verbal modality.

This latter finding could be seen as a weakness, and there is certainly a lot more work to be done; for example, considering the participants' own views of the intervention and its' impact. We would suggest, however, that the picture may be more complex, especially if the most desirable learning outcome of this (indeed, arguably any) coach development intervention is the change in behaviour. For example, given that expertise rather than satisfaction of competency standards is our ultimate aim (cf. Collins et al. 2015), levels of critical reflection are an important precursor. What we still need to know, however, is exactly what levels of criticality (as operationalised here by the methodology of Hatton and Smith, 1995) are needed to drive appropriate levels of introspection. Finally, and reflecting suggestions offered by Stoszkowski and Collins (2012), it may be that social interactions between coaches (as facilitated by the Flipgrid approach) may represent an alternative or more likely additional tool to generate behaviour change.

These important next steps notwithstanding, the results demonstrate an effective and seemingly student-palatable means of increasing engagement in reflective thinking. We commend the approach to colleagues in higher education and more traditional coach education environments. 


\section{References}

Abrami, P. C., R. M. Bernard, E. M. Bures, E. Borokhovski, and R. M. Tamim. 2011. "Interaction in Distance Education and Online Learning: Using Evidence and Theory to Improve Practice.” Journal of Computing in Higher Education 23 (2-3): 82-103.

Byington, T. 2011. "Communities of Practice: Using Blogs to Increase Collaboration." Intervention in School and Clinic 46: 280-291. doi:10.1177/1053451210395384

Clarà, M. 2015. "What Is Reflection? Looking for Clarity in an Ambiguous Notion." Journal of Teacher Education 66 (3): 261-271. doi: 10.1177/0022487114552028

Collins, D., A. Martindale, V. Burke, and A. Cruickshank. 2015. "The Illusion of Competency versus the Desirability of Expertise: Seeking a Common Standard for Support Professions in Sport." Sports Medicine 45 (1): 1-7. doi: 10.1007/s40279-0140251-1

Costa, A., and B. Kallick. 1993. "Through the Lens of a Critical Friend.” Educational Leadership 51 (2): 49-51.

Courtney, M., and S. Wilhoite-Mathews. 2015. "From Distance Education to Online Learning: Practical Approaches to Information Literacy Instruction and Collaborative Learning in Online Environments." Journal of Library Administration 55 (4): 261277. doi: 10.1080/01930826.2015.1038924

Cushion, C., and R. C. Townsend, R. 2019. “Technology-enhanced Learning in Coaching: A Review of Literature.” Educational Review 71 (5): 631-649. doi: $10.1080 / 00131911.2018 .1457010$

Dymoke, S., and J. Harrison. 2008. Reflective Teaching and Learning: A Guide to Professional Issues for Beginning Secondary Teachers. London: Sage. 
Entwistle, N. J., and E. R. Peterson. 2004. "Conceptions of Learning and Knowledge in Higher Education: Relationships with Study Behaviour and Influences of Learning Environments.” International Journal of Educational Research 41: 407-428.

Freeman, W., and C. Brett. 2012. "Prompting Authentic Blogging Practice in an Online Graduate Course.” Computers and Education 59 (3): 1032-1041. doi:

10.1016/j.compedu.2012.03.019

Gould, D., J. Nalepa., and M. Mignano. 2019. “Coaching Generation Z Athletes.” Journal of Applied Sport Psychology Advance Online Publication. doi: $10.1080 / 10413200.2019 .1581856$

Gunawardena, C. N. 1995. "Social Presence Theory and Implications for Interaction and Collaborative Learning in Computer Conferences." International Journal of Educational Telecommunications 1 (2): 147-166.

Hatton, N., and D. Smith. 1995. "Reflection in Teacher Education: Towards Definition and Implementation.” Teaching and Teacher Education 11 (1): 33-49.

Kreijns, K., P. A. Kirschner, and W. Jochems. 2003. "Identifying the Pitfalls for Social Interaction in Computer-Supported Collaborative Learning Environments: A Review of the Research." Computers in Human Behavior 19 (3): 335-353.

Leach, L., G. Neutze, and N. Zepke. 2001. “Assessment and Empowerment: Some Critical Questions.” Assessment and Evaluation in Higher Education 26 (4): 293 305. doi: $10.1080 / 02602930120063457$

LeFebvre, L., E. L. LeFebvre, and M. Allen. 2018. "Training the Butterflies to Fly in Formation: Cataloguing Student Fears About Public Speaking." Communication Education 67 (3): 348-362. doi: 10.1080/03634523.2018.1468915

McGrath, J. E., and A. B. Hollingshead. 1993. "Putting the 'Group' back in Group Support Systems: Some Theoretical Issues about Dynamic Processes in Groups with 
Technological Enhancements.” In Group Support Systems: New perspectives, edited by L. M. Jessup, 78-96. New York: MacMillan Publishing.

Mennecke, B. E., J. S. Valacich, and C. B. Wheeler. 2000. “The Effects of Media and Task on User Performance: A Test of the Task-Media Fit Hypothesis." Group Decision and Negotiation 9: 507-529.

Northrup, P. 2001. “A Framework for Designing Interactivity into Web-Based Instruction.” Educational Technology 41: 31-39.

Nowell, L., J. Norris, D. White, and N. Moules. 2017. “Thematic Analysis: Striving to Meet the Trustworthiness Criteria.” International Journal of Qualitative Methods (December 2017). doi: $10.1177 / 1609406917733847$

Piggott, D. 2015. “The Open Society and Coach Education: A Philosophical Agenda for Policy Reform and Future Sociological Research.” Physical Education and Sport Pedagogy, 20 (3): 283-298.

Resta, P., and T. Laferrière. 2007. “Technology in Support of Collaborative Learning.” Educational Psychology Review 19: 65-83.

Richmond, V. P., J. S. Wrench, and J. C. McCroskey. 2013. Communication Apprehension, Avoidance, and Effectiveness. Boston, MA: Pearson.

Saylor, C. R. 1990. "Reflection and Professional Education: Art, Science, and Competency." Nurse Educator, 15 (2): 8-11.

Schlenker, R. B., and R. M. Leary. 1982. "Social Anxiety and Self-Presentation: A Conceptualization and Model." Psychological Bulletin 92 (3): 641-66.

Schön, D. 1991. The Reflective Practitioner: How Professionals Think and Act. Oxford: Avebury. 
Sins, P. H. M., E. R. Savelsbergh, W. R. van Joolingen, and B. H. A. M. van Hout-Wolters. 2011. "Effects of Face-to-Face versus Chat Communication on Performance in a Collaborative Inquiry Modeling Task.” Computers and Education 56: 379-387.

Smith, B., and K. McGannon. 2018. "Developing Rigor in Qualitative Research: Problems and Opportunities within Sport and Exercise Psychology." International Review of Sport and Exercise Psychology 11 (1): 101-121. doi: 10.1080/1750984X.2017.1317357

Sports Coach UK. 2012. The UKCC Level 1-3 Support Guide. Leeds: Coachwise.

Stoszkowski, J., and D. Collins. 2012. "Communities of Practice, Social Learning and Networks: Exploiting the Social Side of Coach Development." Sport, Education and Society 19 (6): 773-788. doi: 10.1080/13573322.2012.692671

Stoszkowski, J., and D. Collins. 2014. "Blogs: A Tool to Facilitate Reflection and Community of Practice in Sports Coaching?" International Sport Coaching Journal 1 (3): 139-151.

Stoszkowski, J., and D. Collins. 2016. "Sources, Topics and Use of Knowledge by Coaches.” Journal of Sports Sciences 34 (9): 794-802.

Stoszkowski, J., and D. Collins. 2017. "Using Shared Online Blogs to Structure and Support Informal Coach Learning. Part 1: A Tool to Promote Reflection and Communities of Practice?" Sport, Education and Society 22 (2): 247-270.

Stoszkowski, J., D. Collins, and C. Olsson. 2017. "Using Shared Online Blogs to Structure and Support Informal Coach Learning. Part 2: The Participants' View and Implications for Coach Education.” Sport, Education and Society 22 (3): 407-425.

Stoszkowski, J., and D. Collins. 2018. "The Agony and the Ecstasy: Student-Coaches' Perceptions of a Heutagogical Approach to Coach Development.” International Sport Coaching Journal 5 (2): 136-144. 
Straus, S. G. 1997. “Technology, Group Process, and Group Outcomes: Testing the Connections in Computer-Mediated and Face-to-Face Groups.” Human-Computer Interaction 12: 227-266.

Stronach, I., D. Garratt, C. Pearce, and H. Piper. 2007. "Reflexivity, the Picturing of Selves, the Forging of Method." Qualitative Inquiry 13 (2): 179-203. doi: $10.1177 / 1077800406295476$

Thompson, N., and J. Pascal. 2012. "Developing Critically Reflective Practice." Reflective Practice 13 (2): 311-325. doi: 10.1080/14623943.2012.657795

Tsiotakis P., and A. Jimoyiannis. 2016. “Critical Factors Towards Analysing Teachers' Presence in On-line Learning Communities.” The Internet and Higher Education 28: 45-58. doi: 10.1016/j.iheduc.2015.09.002

Van der Linden, J., and P. Renshaw. 2001. Dialogic Learning. Dordrecht: Kluwer Academic Publishers.

Walker, K. 2000. "It's Difficult to Hide It: The Presentation of Self on Internet Home Pages." Qualitative Sociology 23 (1): 99-120.

Wass, R., T. Harland, and A. Mercer. 2011. "Scaffolding Critical Thinking in the Zone of Proximal Development." Higher Education Research and Development 30 (3): 317 328. doi: $10.1080 / 07294360.2010 .489237$

Wenger, E., R. McDermott, and W. M. Snyder. 2002. Cultivating Communities of Practice. Boston: Harvard Business School Press.

Whipp, J. L. 2003. “Scaffolding Critical Reflection in Online Discussions: Helping Prospective Teachers Think Deeply about Field Experiences in Urban Schools." Journal of Teacher Education 54 (4): 321-333. doi:10.1177/0022487103255010 
Yang, S. H. 2009. "Using Blogs to Enhance Critical Reflection and Community of Practice." Educational Technology and Society 12 (2): 11-21. 